\title{
A lightweight approach for providing Location Based Content Retrieval
}

\author{
Authors: Anastasios Zafeiropoulos, Emmanuel Solidakis, Stavroula Zoi, Nikolaos Konstantinou, Panagiotis \\ Papageorgiou, Panagiotis Stathopoulos, Nikolas Mitrou \\ National Technical University of Athens, Heroon Polytechneiou Str., 15773 Zografou, Athens, Greece \\ E-mail: \\ \{tzafeir;nkons\}@cn.ntua.gr; \{esolid;pstath;vzoi;ppapage\}@telecom.ntua.gr;mitrou@softlab.ntua.gr
}

\begin{abstract}
This paper presents a lightweight approach for providing web-based location aware multimedia content retrieval through Java enabled handheld devices. The main distinguishing characteristic of the proposed approach is that it separates the positioning system from the content access mechanisms, while being generic to the selection of the localization technology, i.e. GPS, Bluetooth, etc. Furthermore, it is built as an open, standards-based, modular architecture comprising a core of reusable components and interfaces for supporting different types of services, through web technologies. On-site services are provided through smart phones, which exploit the user's contextual state, mainly defined as end-user location and organization of points of interest. A test case of a museum e-guidance application for Bluetooth enabled smart phones is presented. The test case demonstrates the capacity of the approach to provide location based content retrieval to museum visitors while using their personal phones, without the need for the visitors, or the museum, to acquire and manage specially built user terminals, e.g. PDAs with GPS modules.
\end{abstract}

Keywords: Location Aware Multimedia Content Retrieval, Smart phones, Bluetooth, GPS, Web technologies, Ajax applications.

\section{INTRODUCTION}

A variety of localization and navigation based mechanisms and frameworks are available nowadays, aiming to provide indoor and outdoor location based services to end users. One of their main characteristics is that they are usually tightly coupled to the localization technology, user device, and content access mechanisms and technologies. Thus, specialized hardware and software capabilities are needed to exploit them. Finally, human interaction is needed most times not only to install them but also to support them during their operation. Delays in the significant wide scale adoption of location based content retrieval services can be traced to the aforementioned factors.

Based on the above observations, we present a lightweight approach for providing location aware multimedia content retrieval, through Java enabled handheld devices. The main distinguishing characteristic of the proposed approach is that it separates the positioning system from the content access mechanisms, while being generic to the selection of the radio localization technology. So far, interfaces are provided for Bluetooth and Infrared for indoor environments, as well as for GPS for outdoor environments, while others can be developed, e.g. RFID. Furthermore, it is built as an open, standards-based, modular architecture comprising a core of reusable components and interfaces for supporting different types of services, through web technologies. A basic characteristic of the proposed approach is that it has minimum mobile device requirements, since it operates in all java enabled mobile devices. Therefore, only a Java middlet and a web browser need to be installed in the mobile device, while, in the backend what is required is an HTTP server and CMS system which can be determined independently. The components of the proposed approach ensure modularity, in that different types of services can be easily supported for different groups of users.

After highlighting the most important aspects of this work we summarize the paper structure. First we present the criteria and requirements for designing Location Based Services (LBS) and we briefly compare the existing indoor tracking technologies as well as the existent systems for LBS. Then we present the proposed system architecture focusing on the innovative features of our approach: the Mobile Device Software, the Location to URI Database, the Application's Logic Description and the Redirection Mechanism. At the end a test case application for a museum e-guidance scenario is demonstrated implemented based on the proposed approach.

\section{RELATED WORK}

\section{A. Background information - Criteria and Requirements for Designing LBS}

As described in [4], in order to develop a platform to provide LBS, the following requirements need to be fulfilled: terminal independence, simple user interface, minimal communication over mobile telephone networks, integration of mobile devices, simple integration of existing Internet services, high availability of the services even at high loads, scalability, openness, and low costs. Furthermore, in [3] there are analyzed the design criteria to use when developing location-aware indoor mobile applications. The most important of them are the following: easy navigation through web browsers, navigation feedback and minimal graphical interaction, orientation support in the surrounding environment and minimum redundancy in input commands.

\section{B. Short Comparison of Position Tracking technologies}

Position tracking technologies aim to measure the movement of the mobile terminal. These technologies provide great accuracy, but are limited in terms of geographic coverage. To explicitly localize the users in indoor applications, three recent technologies are mainly 
exploited: WLAN, Bluetooth and Infrared. In [3] advantages and disadvantages of each of them are highlighted. In the next paragraph we provide an overview of these technologies.

WLAN technology allows devices to immediately connect to a LAN. As stated in [3], to locate the position of the users in a building, WLAN is not a so simple solution, because the system has to apply triangulation methods to the data coming from at least three access points near the user. Bluetooth technology is an ad hoc technology that requires no fixed infrastructure and is simple to install and set up. A fundamental Bluetooth wireless technology strength is the ability to simultaneously handle both data and voice transmissions with low power and cost. IrDA protocol of communication supports high data rates and requires line-of-sight contact. But infrared has some drawbacks. Firstly, it rebounds over the surfaces and secondly requires that sender and receiver are aligned. GSM Location Based Services provide personalized services to the subscriber based on their current position. But, as stated in [2], cellular positioning technologies are an opportunistic development rather than the original purpose of cellular networks and consequently cellular technologies are less accurate. Finally, GPS is used from the majority of LBS systems for outdoor tracking environments. The position calculated by a GPS receiver requires the current time, the position of the satellite and the measured delay of the received signal. The position accuracy is primarily dependent on the satellite position and signal delay.

As far as it concerns alternative positioning systems, the use of RFID tags was not discussed as an implementation choice, as experience from relative projects has shown some drawbacks [16], focusing, especially, on the restriction to short-range communication and other issues [17].

\section{Existing Systems for LBS}

In [3], [5], [6] \& [7] many existent applications for LBS are presented (Cyberguide project [8], GUIDE project [10], Hippie/HIPS project [11], CyberAssist project [12], TellMaris project [13], LOL@ System [9], REAL project [14] and Context Sensitive Computing program [15]). The technologies deployed to track the mobile user's location, the accuracy and reliability associated with such measurements and the network infrastructure elements deployed are illustrated.

According to [6], in terms of positioning, roughly half of the systems rely on GPS while a large group of them use infrared beacons. Furthermore, about half of the systems include some means of interacting with the user to determine his position. As far as the architecture is concerned, some systems are based on the client-server paradigm while others are built using interactive applications. The first have the advantage that, given a reliable connection between client and server, they allow easy adoption of multiple clients. The later offer a more decentralized approach, but they may depend on a certain device/platform.

In [18], among others, advanced wireless services are provided in the Athens International Airport based on GPS and WLAN technologies, on a distributed, agentbased architecture. Also, in [19], AVATON, an ambient information system allowing GPS based, location-aware, interactive guidance inside the area of the Aegean Volcanic Arc is presented. In AVATON, a guidance session includes complex content retrieval composed of geographical, historical and geological information and can be realized both on-site through handheld devices, and through the Internet and TV channels. AVATON architecture is built based on the client-server paradigm and Web technologies, with the focus being on serving multiple communication channels through a common content server.

Based on the experience acquired from the above frameworks, we present a lightweight approach for providing Location Based Content Retrieval. In accordance with the approach described in next sections, we have developed a museum e-guidance system application, as it is described in [20].

\section{Approaches for providing $L B S$}

In order to provide Location Based Services (LBS) one or more location tracking methods can be used to determine the position of user equipment [1]. Location tracking techniques in general operate in two steps — the first concerns signal measurements and the second location estimate computation based on the above measurements. These, may be carried out either by the user equipment or by the telecommunications network.

In [2], a flexible framework for using Positioning Technologies in LBS is presented, in which the roles of the positioning system and the client side application are separated. In this framework a positioning technology should provide both rapid and accurate location information. With a hybrid approach the framework can operate both indoors/outdoors transparently to the user, handover between positioning technologies and select successfully the appropriate positioning system. But, this approach is based only on satellite and cellular positioning technologies. Limitations are imposed to the user, especially in indoors systems, to use GSM technologies which are less accurate. Furthermore, the components that should have such an implementation are not described adequately. In the work described in this paper, a hybrid approach is provided that is modular in all its interfaces and to a great extent independent from the localization technology and the mobile device used.

\section{The LightWeight APPROACH - THE SYSTEM LEVEL ARCHITECTURE}

In this section the proposed approach for providing webbased location aware multimedia content retrieval through Java enabled devices is presented. In order to avoid system lock-in and to reduce development costs our approach is based on open standards and open source components, where possible. Furthermore, independence from the underlying hardware and software, by selecting generic widespread approaches, is provided. For the rest of the paper, with the term "point of interest" we refer to each point, which triggers a content request to be returned to the mobile device.

\section{A. High Level Features}

The main distinguishing feature of the proposed approach, in comparison with the implementations reported in previous sections, is first of all, that the various system 
components are designed and implemented in a modular manner in order to select the most appropriate in each specific installation. This modularity regards:

- The separation of the positioning system from the content access mechanisms.

- The selection of the localization radio mechanism, accordingly with the implementation site.

- The selection of the terminal mobile device. The only requirement for the mobile device is to be Java enabled, something very common in the majority of the new generation mobile phones, PDAs e.t.c. The content is being presented to the device through any available web browser.

- Independence from the underlying hardware infrastructure. The entire system is based on Web technologies, which can be deployed easily in any server.

- Independence from the content being available to the end-user. All the content is available through the content management server, and can be renewed dynamically without any change and any intervention to our system.

In addition, the entire process is transparent to the user. In opposition with other LBS frameworks, no input is requested from the end-user. He just receives the appropriate content in his display, in correspondence with his position. The system is designed so that all the amount of data is transferred through the local communication infrastructure.

\section{B. Core elements of the System Architecture}

In this section, we describe the core elements of the proposed system architecture. The different components of our approach are the end-user terminals, the backend platform components and the communication infrastructure. The main characteristics of these components are the following:

- Localization technologies such as Bluetooth, Infrared or GPS. These can be integrated to the user device or provided as a separate hardware module.

- $\quad$ The communication network, which can be any IP enabled access network, e.g. a WLAN network, GPRS or $3 \mathrm{G}$ network.

- The Terminal Module, that runs on the mobile device. This is essentially a J2ME middlet which implements the generic localization approach, enables location tracking by using the terminal localization device interfaces, and communicates with the server-side component, which translates physical location to content URI. It also provides the appropriate Graphical User Interfaces.

- $\quad$ The localization server for providing association between location information given by the user terminal and a specific point of interest. The association can be performed independently of the localization mechanism selected - the content is completely orthogonal to the localization mechanism and can be renewed easily. This kind of association is URI-based, because each point of interest corresponds to a specific content URI in the server.

- $\quad$ The content related to each point of interest which becomes automatically available to the user terminal by the content management system (CMS), through the IP access network.

- The redirection mechanism, which is based on AJAX technologies [21]. As far as the terminal-side is concerned it has to support JavaScript. Otherwise, the redirection is implemented through periodic HTTP refreshes to the server-side.

The core elements of the proposed system architecture and the way they are connected are shown in Figure1.

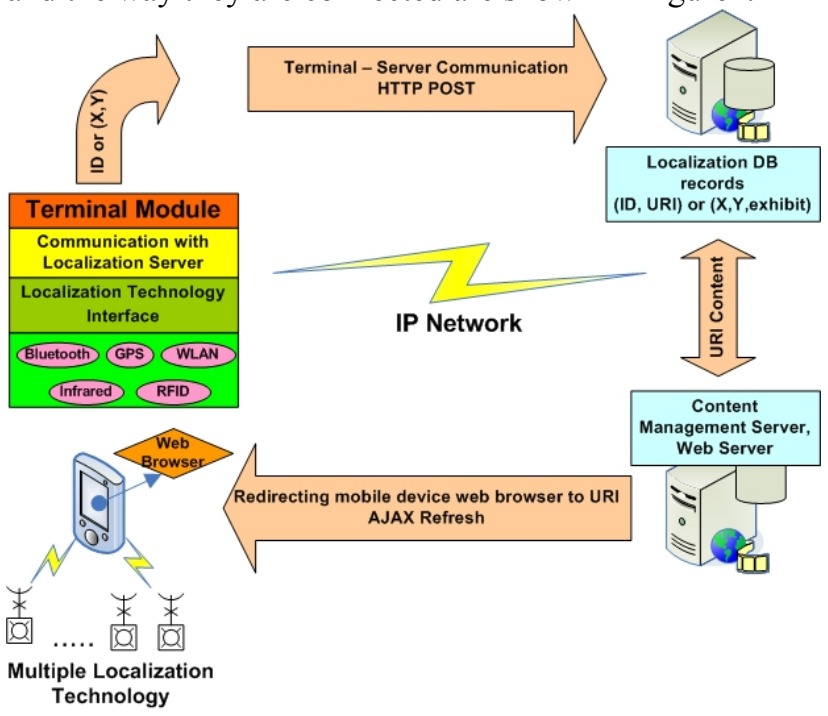

Figure1: Core System Elements

\section{Operational Scenario}

In the following section we describe the simple steps that a user has to follow in order to access the application and the subsequent interaction among the system modules.

1. The user downloads the middlet from the IP network using a Web interface, and installs it in his mobile device.

2. Then, his mobile device scans repeatedly, in a defined time space, for points of interest through the appropriate localization technique.

3. Every time the middlet scans a new point of interest, it communicates with the server and informs it about the new data. The communication is done via HTTP POST messages.

4. The server holds information about the nearest point of interest at which each user is every moment and consequently sends to the terminal device all the related content.

5. The user needs only to open a web browser to his terminal device in a default page and, through the redirection mechanism he will be redirected to the page with the content of the selected point of interest. This default page can be the same page that the user has downloaded the initial middlet.

6. Each time a new localization trigger is received, the user will be redirected to the content of the new point of interest.

Special attention has to be given on the right placement of the points of interest. In case of redundant points that need to be identified within a limited space, only one localization interface is installed. The corresponding web page with this interface is displayed on the user terminal display, with multiple thumbnail images for the points of interest, to allow the user to select the desired web page. 
By this procedure, the offered application is reliable in all cases.

\section{SYSTEM IMPLEMENTATION}

\section{A. Mobile Device Software-Terminal Module}

As described earlier, in order to search and select the desired point of interest, we have developed a Java middlet that can be installed easily in any mobile device that supports Connected Limited Device Configuration CLDC and Mobile Information Device Profile - MIDP of Java Platform Micro Edition - J2ME. The development of this middlet has been accomplished with Netbeans IDE 5.5 extended with the Mobility Pack for CLDC/MIDP, while the simulations were implemented with Sun Java Wireless Toolkit for CLDC 2.5.

There is a configuration menu in the start form of the middlet, where each user can select language, audio and video options and complexity level for the application.

In case of indoor environments, the end-user performs initially a search for localization interest points -that exist within the scope of the terminal- and the ID's of all the detected interest points are returned. After the completion of this search, a new form is opened in the middlet where all the points of interest found are written. Then the nearest point of interest is automatically selected and relevant information is being sent to the server through an HTTP post. This information regards the IP address of the mobile device, the unique ID of the point of interest, the exact time of the request and a session variable being generated at the start of the middlet. The session variable is used to discriminate the use of the application from different users in the same terminal. Then the thread of the middlet is being restarted, after a definite time space, and a new scan is conducted. Furthermore action is taken only in the occasion where a new point of interest is selected. In this occasion a new HTTP post is being done to the server with information about the new point of interest (the session variable remains the same).

In case of tracking technologies that are based on user coordinates (e.g. GPS), the localization mechanism sends an exact geographic coordinate instead of the ID. An extra module is implemented in the server which converts specific coordinates to unique IDs of points of interest. All this information is transmitted through the IP access network and is stored in the localization database.

\section{B. The Location to URI Database-Localization Server}

The database of the entire system is set-up on the server. The database holds information about the points of interest, the devices that executed the application and information regarding the association between them. Extra data are also saved, correlating each point of interest with a URI, which leads to the specific content for this point of interest. Each time the Java middlet is started, the database is updated with the new information sent from the mobile device.

We have defined various actors for different users in our database scheme. The knowledge level and the level of specialisation of every user are stored. Different URIs are saved according to the native language, the skills and the experience of the end-users.

\section{The Application's Logic Description}

As far as the application logic is concerned, our application takes input from all the terminal devices through the middlet running on each of them, updates the database with the new user and localization information, queries the database for the content of the new point of interest, and it posts back a proper URI with the new point of interest info. Every location corresponds to a URI at the CMS server, as described earlier, - featuring some content -static or dynamically generated. Almost everything is controlled from the server, increasing a lot the easiness of use for the end-user. Changes in the content don't affect our application, rendering it content independent.

\section{The Redirection Mechanism}

After the initial installation of the middlet on the endusers terminals, their browsers point to the web-pages describing the corresponding nearest point of interest. Ajax technology is used in order for the terminals to remain active, waiting for the middlet to update the database with the next point of interest. As soon as the middlet inserts a new entry in the database, new content is pushed to the browser by the use of Ajax. The web-page is refreshed asynchronously pointing to URI of the new point of interest. In this way the web pages do not have to be reloaded periodically. This is done only in case where the terminal device doesn't support JavaScript. The intent is to make web pages more responsive by exchanging small amounts of data with the server behind the scenes, so that the entire web page does not have to be reloaded each time the user requests a change. This is meant to increase the web page's interactivity, speed, and usability. The various system components aforementioned, (presentation, database, multi-device interfaces, etc.) are designed and implemented in a modular manner in order to select the appropriate components in each specific installation. Furthermore, the platform is independent of the underlying hardware infrastructure. Thus the platform supports a variety of communication means for the on-site terminals but also a variety of localization techniques (e.g. GPS, Bluetooth, IrDA or RFID in the future).

\section{THE E-MUSEUM DEMONSTRATION APPLICATION}

A museum e-guidance system application has been developed in the scope of the E-Museum project ${ }^{(*)}$ that utilizes Bluetooth localization technology for the positioning of the museum exhibits and WLAN technology for the IP network communication. Content management functions are built based on an existing CMS, as well as on e-museum specific extensions. The open source Joomla CMS has been selected. In addition, we have developed special templates for mobile devices with the use of the Xe-Media Mobile Template for Joomla. In each request, the server checks the browser that makes the request and accordingly returns the page in the appropriate template.

Based on the proposed architecture, E-museum system can easily support a wide range of services, targeting at different user profiles and terminal devices. The most important services that are provided in the scope of this project are the following: Automatic (push) content retrieval based on visitor proximity to an exhibit or group of exhibits, on demand (pull) content retrieval, creation of 
live linear guidances, registration of visited paths and content of interest for offline usage, content publishing to the web, system management and update of the content base, and electronic guest book.

The E-museum system overview is shown in Figure2.

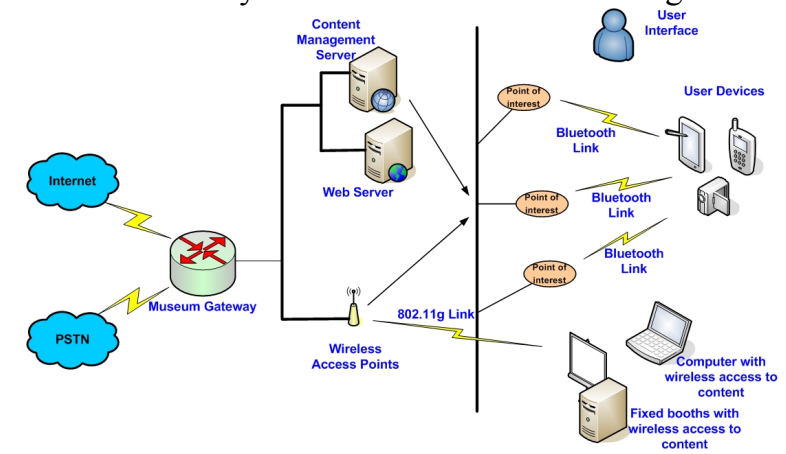

Figure2: E-museum System Overview

As far as the implementation is concerned, we chose to use a Nokia E61 smart phone with Symbian OS, a 3COM 802.11g WLAN access point and a Linux HTTP and MySQL Database Server. A screenshot from the mobile device used is shown in Figure3.

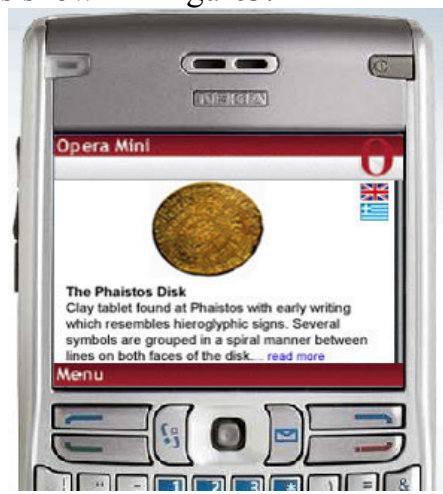

Figure 3: E-museum Screenshot

\section{CONCLUSIONS}

Based on the assumptions that it is desired to allow an application to dynamically select localization technology, the appropriate software and hardware for the backend platform, as well as the terminal mobile device, we have argued that a comprehensive solution should address the challenges of modularity and openness. We therefore proposed an approach for providing Location Based Content Retrieval that attempts to address these issues, while we described an architectural framework for enabling such a system. The application of the framework has been evaluated in a prototype e-museum guidance system, highlighting some of issues involved in the use of location-based services.

Future work will include the support of user-transparent handover process aiming at the selection of the most suitable localization technique, in heterogeneous environments (e.g. indoor-outdoor). Experimental work is currently underway towards evaluating the behaviour of the different localization techniques in several, environments.

\section{ACKNOWLEDGEMENT ${ }^{(*)}$}

Parts of the work presented are funded by the Semantix SA in the framework of the GSRT PAVET-NE research and development action. The authors of this paper would like to thank their colleagues in Semantix S.A. (http://www.semantix.gr) for their contribution and cooperation.

\section{REFERENCES}

[1] Stuart J. Barnes, "Location Based Services", e-Service Journal, 2003.

[2] J. Ranchordas \& A. Lenanghan, "A Flexible Framework for using Positioning Technologies in Location-Based Services", EUROCON 2003..

[3] Carmine Ciavarella \& Fabio Paterno , "Design Criteria for Location-aware, Indoor, PDA applications", Proceedings of Fifth International Symposium on Human Computer Interaction with Mobile Devices and Services, Udine, Italy, 2003, ع $\pi$. L. Chittaro, Lecture Notes in Computer Science, Vol. 2795, 131-144. Berlin: Springer-Verlag.

[4] Sebastian Herden, Arman Mkrtchyan, Claus Rautenstrauch, Andrı Zwanziger \& Michael Schenk, "Personal Information Guide - A Platform with Location Based Services for Mobile Powered Ecommerce", DEXA 2003.

[5] Dillip Mohapatra, Suma S.B, "Survey of Location Based Wireless Services", Personal Wireless Communications, 2005. ICPWC 2005 IEEE International Conference, 2005.

[6] Christian Kray, Jorg Baus, "A Survey of Mobile Guides”, ICPWC 2005.

[7] Todd Simcock, Stephen Peter Hillenbrand \& Bruce H. Thomas, "Developing a Location Based Tourist Guide Application", 2003.

[8] D. A. Abowd, C. G. Atkeson, J. Hong, S. Long, K. R., and M. Pinkerton. Cyperguide: A Mobile Context-Aware Tour Guide. Wireless Networks, 3(5):421-433, 1996.

[9] H. Anegg, H. Kunczier, E. Michlmayr, G. Pospischil, and M. Umlauft.LoL@: designing a location based UMTS application. Elektrotechnik und Informationstechnik, 119(2):48-51, 2002.

[10] K. Cheverst, N. Davies, K. Mitchell, A. Friday, and C. Efstratiou. Developing a Context-aware Electronic Tourist Guide: Some Issues and Experiences. In Proceedings of the 2000 Conference on Human Factors in Computing Systems (CHI-00), pages 17-24, New York, 2000. ACM Press.

[11] R. Oppermann and M. Specht. A Context-Sensitive Nomadic Exhibition Guide. In Second Symposium on Handheld and Ubiquitous Computing - HUC2K, pages 127-149, Berlin, 2000. Springer.

[12] CARC. Web page of the Cyber Assist Research Center (CARC). http://www.carc.aist.go.jp, 2002.

[13] C. Kray, K. Laakso, C. Elting, and V. Coors. Presenting route instructions on mobile devices. In W. L. Johnson, E. Andr'e, and J. Domingue, editors, Proceedings of IUI 03, pages 117-124, Miami Beach, FL, 2003. ACM Press.

[14] J. Baus, C. Kray, and A. Kruger. Visualization of route descriptions in a resource-adaptive navigation aid. Cognitive Processing, 2(2-3):323-345, 2001.

[15] Dey, A. K. and Abowd, G. D. (2000) In Proceedings of the 2nd International Symposium on Handheld and Ubiquitous Computing (HUC2K)Bristol, UK, pp. 172-186.

[16] Luyten, K. and Coninx, K. ImogI: Take Control over a Context Aware Electronic Mobile Guide for Museums. HCI in Mobile Guides, 13 September 2004, University of Strathclyde, Glasgow.

[17] C. Floerkemeier and M. Lampe. Issues with RFID Usage in Ubiquitious Computing Applications. In Pervasive Computing, pages 188-193, 2004.

[18] Y. Wang, L. Cuthbert, Francis J. Mullany, P. Stathopoulos, V. Tountopoulos, M. Senis, "Exploring Agent-based Wireless Business Models and Decision Support Applications in an Airport Environment", Journal of Telecommunications and Information Technology, no. 3/2004

[19] P. Kalliaras, A. D. Sotiriou, S. Zoi, P. Papageorgiou, J. N. Karigiannis, N. Farantouris, N. Mitrou "The Avaton Architecture: Location-Based Multimedia Services For Tourists" in ITI 2nd International Conference on Information \& Communication Technology (ICICT 2004) 6, 7 December 2004, Cairo - EGYPT

[20] Panagiotis Stathopoulos, Stavroula Zoi, Nikolaos Konstantinou, Emmanuel Solidakis, Christos Basios, Tasos Zafeiropoulos, Panagiotis Papageorgiou, Nikolas Mitrou, "A content management system for providing museum visitors personalised audiovisual information", $3^{\text {rd }}$ International Conference of museology, ICOMAVICOM, 5-9 June 2006

[21] Jesse Garrett, "Ajax: A New Approach to Web Applications", http://www.adaptivepath.com/publications/essays/archives/000385. php, 2005. 